\title{
Transcriptome of the Arabidopsis thaliana Chernobyl ecotype seedlings: simulating of the space radiation action and microgravity
}

\author{
Kazakova E.A. ${ }^{1 *}$, Podlutskii M.S. ${ }^{1}$, Moiseev A.S. ${ }^{2}$, Kazakov E.I. ${ }^{2}$, Saburov V.O. ${ }^{2}$, \\ Babina D.D. ${ }^{1}$, Podobed M.Yu. ${ }^{1}$, Volkova P.Yu. ${ }^{1}$ \\ ${ }^{1}$ Russian Institute of Radiology and Agroecology, Obninsk, Russia \\ ${ }^{2}$ A. Tsyb MRRC, Obninsk, Russia \\ * email: elisabethafeb19@gmail.com
}

The problem of search for biological mechanisms of plant response to extreme space conditions, such as ionizing radiation and microgravity, is important for space biology in the aspect of long-term space missions to Mars. We exposed Arabidopsis thaliana seedlings of the Chernobyl ecotype (ChE) and wild type Col-8 to radiation combined with simulated microgravity, as well as to the microgravity and protons separately. An energy of protons was $100 \mathrm{MeV}$, for each sample the total absorbed dose was $10 \mathrm{~Gy}$, rotation speed of the 3D-clinostate was $60 \mathrm{rpm}$. The analysis of the seedlings transcriptome was performed on the Illumina NovaSeq 6000 platform; more than 990 million reads were obtained. In seedlings of the $\mathrm{ChE}$ and wild type exposed to simulated space conditions, 44 and 47 up-regulated differentially expressed genes (DEGs) $\left(\log _{2} \mathrm{FC}\right.$ $>2$, FDR adjusted $p$-value $<0.05$ ) were detected, compared with control seedlings of $\mathrm{ChE}$ and Col-8 respectively. In exposed ChE seedlings, 8 unique DEGs relatively to Col8 were identified associated with DNA repair, replication, and protein metabolism. An analysis of the functional GO enrichment for DEGs in $\mathrm{ChE}$ exposed to proton irradiation and clinostating revealed the enriched terms associated with response to ionizing radiation, DNA repair, and cell cycle; for Col-8, the terms were also associated with the same processes. At the same time, when comparing the genotypes of ChE and Col-8, 165 up-regulated and 206 down-regulated DEGs were revealed in ChE relative to Col8. The results of the work allowed us to identify and compare the most probable molecular pathways that respond to the simulated outer space conditions in the seedlings of the Chernobyl ecotype and wild type A. thaliana, which in the future will make it possible to obtain plants with increased stress resistance to space environmental conditions.

Acknowledgements: Supported by RSF (No. 20-74-00101). 